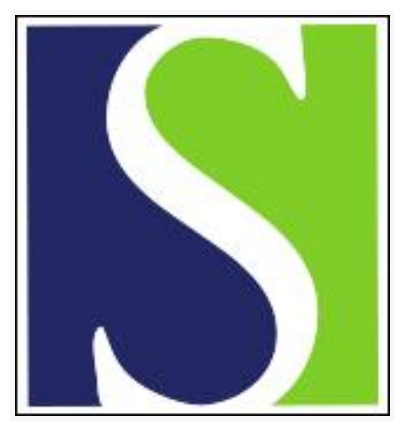

Scand J Work Environ Health 2001;27(6):388-394

https://doi.org/10.5271/sjweh.631

Issue date: Dec 2001

Do psychosocial strain and physical exertion predict onset of low-back pain among nursing aides?

by Gonge H, Jensen LD, Bonde JP

Affiliation: Department of Occupational Medicine, Aarhus Kommunehospital, Nørrebrogade 44, 8000 Aarhus C, Denmark. henrik@gonge.dk

Refers to the following texts of the Journal: 1998;24(6):449-464 1998;24(6):465-472 1997;23(4):243-256 1995;21(6):435-439 1999;25 suppl 4:31-35

The following article refers to this text: 2012;38(3):282-290

Key terms: acute pain onset; case-crossover study; longitudinal study; low-back pain; nursing aide; physical exertion; psychosocial strain; stress; time pressure

This article in PubMed: www.ncbi.nlm.nih.gov/pubmed/11800326

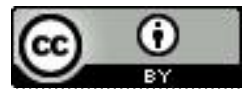




\title{
Do psychosocial strain and physical exertion predict onset of low-back pain among nursing aides?
}

\author{
by Henrik Gonge, PhD, 'Lone Donbæk Jensen, MD, ${ }^{2}$ Jens Peter Bonde, PhD²
}

\begin{abstract}
Gonge $\mathrm{H}$, Jensen LD, Bonde JP. Do psychosocial strain and physical exertion predict onset of low-back pain among nursing aides? Scand J Work Environ Health 2001;27(6):388-394.
\end{abstract}

\begin{abstract}
Objectives The aim of this study was to investigate psychosocial factors and physical exertion at work in relation to the onset of low-back pain.

Methods The study was carried out as a case-crossover investigation of nursing aides caring for the elderly. Cases were identified among 157 nursing aides over a period of 2 years. Psychosocial factors, physical exertion, and low-back pain were reported daily in diary questionnaires over three consecutive days at work, repeated in six periods of 3 days. For each subject, case observations were identified as pain onset from one day to the next and matched with reference observations with no pain onset from the same person. Prospective data collection allowed analyses to be conducted with and without a lag in time between exposure and pain onset.

Results The results of the analyses with time lag (longitudinal) did not support the hypothesis that psychosocial and physical strain from 1 day of work predicts pain onset the following day. However, physical exertion, stress, and, to some extent, time pressure were associated with pain on the day of onset.

Conclusion The effect period, if any, of exposure to physical exertion, stress, and time pressure on the onset of acute low-back pain is considered to be less than 24 hours.
\end{abstract}

Key terms acute pain onset, case-crossover, longitudinal, stress, time pressure.

Female nursing aides have a high prevalence of lowback pain when compared with other nursing personnel $(1,2)$ or with women in the general population (1). It is agreed that the etiology of low-back pain is multifactorial (3), including both physical load and psychosocial factors in relation to individual functional capacity (3, 4). However, reviews reveal that the significance of psychosocial factors is inconsistent $(1,3-5)$ and, further, that previous research often lacks a distinction between acute and chronic pain.

Several studies have suggested that psychosocial factors such as stress (6-8), strain (interaction of high demands and low control) (9-11), low support from superiors (12), feeling of need to slow down (13), perception of permanent work overload (14), and time pressure and troublesome patients with dementia symptoms (7) contribute to the etiology of low-back pain among nursing aides (auxiliary nurses, assistant nurses, practical nurses, home care workers). Other studies indicate that the impact of psychosocial factors gains significance in interaction with measures of physical exertion $(10,11,15,16)$.

Other than the aforementioned cross-sectional studies, to our knowledge, only a limited number of longitudinal $(17,18)$ and case-referent $(2)$ studies focusing on nursing aides has been published. These studies do not suggest that psychosocial factors are causal in relation to low-back pain.

There is a need for studies that test earlier findings in appropriate designs that allow for the inference of causality. Furthermore, designs should be sensitive to fluctuations that reflect the episodic nature of low-back pain. In our present study incidents of acute onset or the aggravation of low-back pain were investigated with a

Department of Occupational Medicine, University Hospital of Aarhus and Institute of Psychology, University of Aarhus, Aarhus, Denmark.

2 Department of Occupational Medicine, University Hospital of Aarhus, Aarhus, Denmark.

Reprint requests to: Dr Henrik Gonge, Department of Occupational Medicine, Aarhus Kommunehospital, Nørrebrogade 44, 8000 Aarhus C, Denmark. [E-mail: henrik@gonge.dk] 
case-crossover design $(19,20)$, which has recently been recommended for studying low-back pain $(21,22)$. Using a case-crossover design with prospective data collection, we set out to examine whether perceived psychosocial or physical strain predicts or, alternatively, is associated with the onset of low-back pain. It was hypothesized that increased psychosocial strain or physical exertion (i) predicts pain onset the following day or (ii) is associated with pain onset the same day.

\section{Subjects and methods}

\section{Population}

All employees in three Danish municipalities working in residential homes and home care for elderly or handicapped people were invited to participate in the study. In Denmark, care for the elderly is mainly organized in centers where the nursing personnel provide home and residential care, including a variety of worktasks primarily concerned with care but also domestic tasks. It was underlined that participation was not dependent on previous incidents of low-back pain. Eighty-nine percent of all the invited employees consented to participate, and $179(85 \%)$ stayed in the program to complete the 2-year longitudinal study. Of these, 157 female home care workers and nursing aides performed job tasks relevant for inclusion in this study. The age of the 157 participants ranged from 18 to 64 (mean 44.5, SD 9.5) years, while seniority within care work ranged from 1 to 42 (mean 14.7, SD 7.8) years.

\section{Data collection}

Data were collected six times by diary with intervals of approximately 3 months. In each diary period of 3 consecutive workdays, data were recorded through self-re- ports. Identical questions were repeated on each day of diary recording, and the participants were instructed to report at the end of each workday with that day in mind. The first set of diaries was handed out personally to each participant, followed by a short individual, oral instruction, while subsequent sets of diaries were mailed. Altogether $728(77 \%)$ out of 942 potential sets of diaries were received from the 157 participants. The average number of diary sets per subject was 4.6 (1st round 141 , 2nd 137, 3rd 128, 4th 116, 5th 109 and 6th 97). Incomplete diary recording was ascribed to absence due to training or sickness, as well as to a general decline in the response rate over time, which was partly attributable to retirement but which was also caused by other unknown reasons.

\section{Measurement of exposure}

The work-related risk factors considered for this study were time pressure, stress, help (support), appreciation from clients, conscience about quality of the work, and the total physical and total mental exertion from care work (table 1). High scores on Likert scales ranging from 0 to 10 for time pressure and stress were expected to constitute increased risk, while low scores for help, appreciation, and conscience could imply an increase in risk. Physical and mental exertion was reported on modified RPE (rating of perceived exertion) scales ranging from 0 to 14 (23). Correlation coefficients between the independent variables are given in table 2 . The strongest correlations ( $r)$ were time pressure-stress $(r=0.72)$ and physical-mental exertion $(\mathrm{r}=0.54)$.

\section{Measurement of low-back pain}

The subjects were asked to "Indicate your low-back pain right now" on a Likert scale ranging from " $0=$ none at all" to " $10=$ worst possible" (table 1). The independent

Table 1. Independent and dependent variables. (RPE = rating of perceived exertion)

\begin{tabular}{|c|c|}
\hline Variables & Scales \\
\hline Time pressure: "Estimate the time pressure at work today during the entire day." & Likert scale ranging from $0=$ very little to $10=$ very, very much \\
\hline Stress: "How much stress have you felt at work today?" & Likert scale ranging from $0=$ no stress to $10=$ very high stress \\
\hline Help: "How much help have you received while doing your work today?" & Likert scale ranging from $0=$ no help to $10=a$ lot of help \\
\hline $\begin{array}{l}\text { Appreciation: "How much appreciation from the clients have you experienced } \\
\text { today?" }\end{array}$ & $\begin{array}{l}\text { Likert scale ranging from } 0=\text { no appreciation to } 10=\text { a lot of } \\
\text { appreciation }\end{array}$ \\
\hline Conscience: "How is your conscience about the quality of the work today?" & $\begin{array}{l}\text { Likert scale ranging from } 0=\text { bad conscience to } 10=\text { good } \\
\text { conscience }\end{array}$ \\
\hline Physical exertion: "Estimate the total physical exertion from the carework today." & $\begin{array}{l}\text { Modified RPE scale ranging from } 0 \text { to } 14 \text { with } 1=\text { very, very light } \\
\text { to } 13=\text { very, very hard }\end{array}$ \\
\hline Mental exertion: "Estimate the total mental exertion from the carework today." & $\begin{array}{l}\text { Modified RPE scale ranging from } 0 \text { to } 14 \text { with } 1=\text { very, very light } \\
\text { to } 13=\text { very, very hard }\end{array}$ \\
\hline Low-back pain: "Indicate your low-back pain right now." & Likert scale ranging from $0=$ none at all to $10=$ worst possible \\
\hline
\end{tabular}


variables correlated only weakly with the reported lowback pain (table 2).

The dependent variable of this study, onset, is a measure of the development or aggravation of acute, low-back pain. Onsets were derived by calculating the differences in low-back pain from one day to the next in each set of diaries (eg, pain onset from the 1st day to the 2 nd or from the 2 nd day to the 3 rd). The magnitude of the onset was based solely on the differences in the scores independently of the actual score on the Likert scale. An increase from 0 to 1 was categorized as " 1 ", while an increase from 2 to 5 was categorized as " 3 ". Therefore the term "onset" was a measure of pain increase which did not presuppose the participant to be pain free at the outset.

\section{Data analysis}

Data were analyzed using a case-crossover approach. Incidents of reported low-back pain onset were identified as cases. The exposure status of the cases at the time of low-back pain onset was compared with the exposure status for that same individual in two reference periods without pain onset (19).

Cases. The cases of onset of low-back pain were categorized into the following levels by the magnitude of onset: onset 1 (81 cases), onset 2 (36 cases), and onset $\geq 3$ (44 cases). For each participant, only one case of pain onset per level of onset magnitude was included to ensure the independence of the observations.

References. The ideal for identifying reference observations was considered to be neutral pain onset indicated by magnitude " 0 ", but, to obtain a sufficient number of referents, a slight decrease indicated by " -1 " was also accepted.

Matching case and reference observations. For a case to be included, preferably two and at least one reference observation should have been available from the same

Table 2. Spearman correlation coefficients for psychosocial factors, physical and mental exertion, and low-back pain.

\begin{tabular}{lrrrrrrr}
\hline & 1 & 2 & 3 & 4 & 5 & 6 & 7 \\
\hline 1. Time pressure & - & & & & & & \\
2. Stress & 0.72 & - & & & & & \\
3. Help & 0.19 & 0.25 & - & & & & \\
4. Appreciation & -0.11 & -0.15 & -0.03 & - & & & \\
5. Conscience & -0.37 & -0.43 & -0.08 & 0.35 & - & & \\
6. Physical exertion & 0.46 & 0.42 & 0.21 & -0.11 & -0.19 & - & \\
7. Mental exertion & 0.41 & 0.44 & 0.13 & -0.12 & -0.23 & 0.54 & - \\
8. Low-back pain & 0.23 & 0.26 & 0.18 & -0.10 & -0.16 & 0.27 & 0.12 \\
\hline
\end{tabular}

person. When a participant presented more than one case observation, cases having reference observations with identical pain scores at the outset and neutrality in pain development were preferred before the random selection.

Subsequently, to eliminate bias due to differences in actual pain level, reference observations were selected so as to have the same pain score indicated on the Likert scale on the day of outset as the case observation. While identical pain scores was the ideal, reference observations were tolerated to differ \pm 1 in the pain score when compared with the case in order not to restrict the number of referents. Reference observations matching the cases were selected in the following order: (i) on the basis of neutrality of onset ( 0 preferred to -1 ), (ii) identical actual pain score ( 0 preferred to \pm 1 ), and, finally, (iii) chance. As a consequence of this rather restrictive selection procedure, a limited number of cases presented only one reference observation (onset 1: 10 observations, onset 2: 2 observations and onset $\geq 3: 8$ observations), but were nevertheless preserved in the data set. For each person, the order of the case and observations was arbitrary (eg, the references did not necessarily precede the case, as the reference material was too small to implement such a criterion of specific order).

We used conditional logistic regression to analyze relations between the independent and dependent variables of the case and reference observations for each subject. Data from each subject were analyzed as a stratum consisting of matched triplets. The analyses were stratified by level of pain onset (eg, onset 1, onset 2, and onset $\geq 3$ ). All the independent variables (time pressure, help, stress, appreciation, conscience, and physical and mental exertion) were entered into the univariate analyses separately. Odds ratios and asymptotic 95\% confidence intervals $(95 \% \mathrm{CI})$ for a unit increase in each of the independent variables were calculated. Hypothesized effects on pain onset are indicated by odds ratios above 1 for time pressure, stress, physical exertion, and mental exertion and by odds ratios of $<1$ for help, appreciation, and conscience. These procedures were repeated twice to conduct analyses with and without time lag. Independent variables reported on the day preceding pain onset were entered into the analyses with time lag (longitudinal), while analyses without time lag (cross-sectional) were based on reports from the day of pain onset.

In addition, physical and mental exertion were entered into a bivariate conditional logistic regression analysis to allow further exploration of the cross-sectional data. Each separate conditional logistic regression analysis met the convergence criteria defined for this SAS (statistical analysis system) procedure (24), while attempts to enter all the independent variables into a multivariate analysis failed to meet the criteria. 
Table 3. Unadjusted average levels of work-related risk factors by magnitude of low-back pain onset — data without and with time lag between the report of exposure and onset. ${ }^{a}$

\begin{tabular}{|c|c|c|c|c|c|c|c|c|c|c|c|c|c|c|}
\hline \multirow{4}{*}{ Risk factor } & \multicolumn{14}{|c|}{ Magnitude of low-back pain onset } \\
\hline & \multicolumn{6}{|c|}{ Low onset 1} & \multicolumn{4}{|c|}{ Medium onset 2} & \multicolumn{4}{|c|}{ High onset $\geq 3$} \\
\hline & \multicolumn{2}{|c|}{$\begin{array}{l}\text { Grand mean a } \\
(\mathrm{N}=157)\end{array}$} & \multicolumn{2}{|c|}{$\begin{array}{l}\text { Cases } \\
(\mathrm{N}=81)\end{array}$} & \multicolumn{2}{|c|}{$\begin{array}{l}\text { References } \\
(\mathrm{N}=162)\end{array}$} & \multicolumn{2}{|l|}{$\begin{array}{l}\text { Cases } \\
(\mathrm{N}=36)\end{array}$} & \multicolumn{2}{|c|}{$\begin{array}{l}\text { References } \\
(\mathrm{N}=72)\end{array}$} & \multicolumn{2}{|l|}{$\begin{array}{l}\text { Cases } \\
(\mathrm{N}=44)\end{array}$} & \multicolumn{2}{|c|}{$\begin{array}{l}\text { References } \\
(\mathrm{N}=88)\end{array}$} \\
\hline & Mean & SD & Mean & SD & Mean & SD & Mean & SD & Mean & SD & Mean & SD & Mean & SD \\
\hline Time pressure & 3.7 & 2.7 & & & & & & & & & & & & \\
\hline Without time lag & & & 4.1 & 2.5 & 4.0 & 2.7 & 4.4 & 2.5 & 4.4 & 2.3 & 4.6 & 2.8 & 3.7 & 2.7 \\
\hline With time lag & & & 3.9 & 2.6 & 4.1 & 2.8 & 4.1 & 2.8 & 4.8 & 2.7 & 4.1 & 2.4 & 4.1 & 2.8 \\
\hline Stress & 2.3 & 2.4 & & & & & & & & & & & & \\
\hline Without time lag & & & 2.8 & 2.4 & 2.9 & 2.6 & 3.4 & 2.5 & 3.3 & 2.5 & $3.7^{*}$ & 3.0 & $2.8^{*}$ & 2.6 \\
\hline With time lag & & & 2.4 & 2.2 & 2.6 & 2.5 & 3.1 & 2.4 & 3.2 & 2.6 & 2.2 & 2.3 & 2.6 & 2.8 \\
\hline Help & 3.0 & 2.9 & & & & & & & & & & & & \\
\hline Without time lag & & & 3.0 & 2.8 & 3.1 & 2.8 & 4.1 & 2.8 & 3.7 & 2.5 & 3.3 & 3.0 & 3.1 & 3.2 \\
\hline With time lag & & & 2.6 & 2.6 & 3.0 & 2.9 & 4.8 & 3.1 & 4.0 & 2.7 & 3.5 & 3.3 & 2.8 & 3.0 \\
\hline Appreciation & 6.4 & 2.3 & & & & & & & & & & & & \\
\hline Without time lag & & & 6.2 & 2.4 & 6.1 & 2.5 & 6.8 & 1.9 & 6.3 & 2.5 & 6.3 & 2.2 & 6.1 & 2.2 \\
\hline With time lag & & & 6.2 & 2.5 & 6.2 & 2.3 & 6.6 & 1.9 & 6.3 & 2.4 & 6.1 & 1.9 & 6.3 & 2.2 \\
\hline Conscience & 7.6 & 2.1 & & & & & & & & & & & & \\
\hline $\begin{array}{l}\text { Without time lag } \\
\text { With time lag }\end{array}$ & & & $\begin{array}{l}7.4 \\
7.5\end{array}$ & $\begin{array}{l}2.3 \\
1.8\end{array}$ & $\begin{array}{l}7.2 \\
7.1\end{array}$ & $\begin{array}{l}2.2 \\
2.4\end{array}$ & $\begin{array}{l}7.2 \\
7.3\end{array}$ & $\begin{array}{l}2.2 \\
2.2\end{array}$ & $\begin{array}{l}7.1 \\
7.0\end{array}$ & $\begin{array}{l}2.3 \\
2.5\end{array}$ & $\begin{array}{l}7.0 \\
7.7\end{array}$ & $\begin{array}{l}2.6 \\
1.9\end{array}$ & $\begin{array}{l}7.5 \\
7.2\end{array}$ & $\begin{array}{l}2.0 \\
2.5\end{array}$ \\
\hline Physical exertion & 5.6 & 2.5 & & & & & & & & & & & & \\
\hline Without time lag & & & 6.2 & 2.2 & 5.9 & 2.1 & $7.0^{\star}$ & 2.6 & $5.8^{\star}$ & 2.4 & $6.8^{*}$ & 2.3 & $6.0^{*}$ & 2.3 \\
\hline With time lag & & & 5.8 & 2.3 & 6.1 & 2.3 & 6.4 & 2.1 & 6.3 & 2.7 & $5.3^{*}$ & 2.3 & $6.0^{*}$ & 2.6 \\
\hline Mental exertion & 4.7 & 2.6 & & & & & & & & & & & & \\
\hline Without time lag & & & 5.1 & 2.3 & 4.9 & 2.2 & 5.5 & 2.6 & 5.2 & 2.7 & 5.0 & 2.8 & 4.8 & 2.6 \\
\hline With time lag & & & $4.6^{*}$ & 2.4 & $5.1^{*}$ & 2.4 & 5.0 & 2.5 & 5.5 & 2.6 & 4.5 & 2.5 & 4.6 & 2.7 \\
\hline
\end{tabular}

a Number of observations $=2184$.

${ }^{*} \mathrm{P}<0.05$ difference in scores for the case and reference observations analyzed with Wilcoxon signed rank sum tests.

\section{Results}

In table 3, unadjusted average levels of work-related risk factors by magnitude of low-back pain onset are given for the days of pain onset (without time lag), as well as for the day preceding pain onset (with time lag). The analyses of data without time lag indicated that the differences between the scores of the case and reference observations were significant for physical exertion associated with the two highest onset levels, 2 and $\geq 3$, while stress was significant in relation to onset $\geq 3$. Regarding data with time lag, the scores differed for physical exertion in relation to onset $\geq 3$ and mental exertion at the lowest onset level 1. No other differences in the data, with or without time lag, reached significance.

\section{Data without a time lag}

The odds ratios and confidence intervals seen in table 4 support, to some extent, the hypothesized relations. Physical exertion approached significance for onset 1 [odds ratio (OR) 1.13] and reached significance for onset 2 (OR. $1.43,95 \%$ CI 1.08-1.90) and onset $\geq 3$ (OR 1.20, 95\% CI 1.00-1.44). The association between onset $\geq 3$ and stress was significant with an odds ratio of 1.23 (95\% CI 1.00-
Table 4. Crude odds ratios (OR) and 95\% confidence intervals $(95 \% \mathrm{Cl})$ for the risk factors in relation to the onset of low-back pain - univariate conditional logistic regression analyses of data without time lag (cross-sectional). a

\begin{tabular}{|c|c|c|c|c|c|c|}
\hline \multirow[t]{2}{*}{ Risk factor } & \multicolumn{2}{|c|}{$\begin{array}{l}\text { Low onset } 1 \\
(\mathrm{~N}=81)\end{array}$} & \multicolumn{2}{|c|}{$\begin{array}{l}\text { Medium onset } 2 \\
(\mathrm{~N}=36)\end{array}$} & \multicolumn{2}{|c|}{$\begin{array}{l}\text { High onset } \geq 3 \\
(\mathrm{~N}=44)\end{array}$} \\
\hline & $\mathrm{OR}$ & $95 \% \mathrm{Cl}$ & OR & $95 \% \mathrm{Cl}$ & $\mathrm{OR}$ & $95 \% \mathrm{Cl}$ \\
\hline Time pressure & 1.02 & $0.90-1.16$ & 0.99 & $0.73-1.32$ & 1.20 & $0.99-1.47$ \\
\hline Stress & 0.96 & $0.82-1.13$ & 1.05 & $0.79-1.39$ & $1.23^{*}$ & * $1.00-1.51$ * \\
\hline Help & 0.99 & $0.86-1.13$ & 1.12 & $0.88-1.41$ & 1.03 & $0.90-1.18$ \\
\hline Appreciation & 1.03 & $0.89-1.18$ & 1.24 & $0.94-1.63$ & 1.08 & $0.85-1.39$ \\
\hline Conscience & 1.07 & $0.91-1.26$ & 1.01 & $0.77-1.33$ & 0.89 & $0.74-1.06$ \\
\hline Physical exertion & 1.13 & $0.94-1.36$ & $1.43^{\star}$ & * $1.08-1.90^{*}$ & ${ }^{*} 1.20^{*}$ & ${ }^{*} 1.00-1.44^{*}$ \\
\hline Mental exertion & 1.04 & $0.90-1.21$ & 1.08 & $0.86-1.37$ & 1.06 & $0.88-1.27$ \\
\hline
\end{tabular}

1.51), while time pressure approached significance with an odds ratio of 1.20 (95\% CI 0.99-1.47). Conscience about the quality of the work is characterized by decreasing odds ratios, reaching an almost significant odds ratio of 0.89 for onset $\geq 3$.

The univariate analyses without time lag were extended by a bivariate analysis to explore the relative 
Table 5. Risk odds ratios (OR) and 95\% confidence intervals $(95 \% \mathrm{Cl})$ for physical and mental exertion in relation to the onset of low-back pain - bivariate conditional logistic regression analysis of data without time lag (cross-sectional). ${ }^{\text {a }}$

\begin{tabular}{|c|c|c|c|c|c|c|}
\hline \multirow[t]{2}{*}{$\begin{array}{l}\text { Type of } \\
\text { exertion }\end{array}$} & \multicolumn{2}{|c|}{$\begin{array}{l}\text { Low onset } 1 \\
(\mathrm{~N}=81)\end{array}$} & \multicolumn{2}{|c|}{$\begin{array}{l}\text { Medium onset } 2 \\
(\mathrm{~N}=36)\end{array}$} & \multicolumn{2}{|c|}{$\begin{array}{l}\text { High onset } \geq 3 \\
(\mathrm{~N}=44)\end{array}$} \\
\hline & $\mathrm{OR}$ & $95 \% \mathrm{Cl}$ & $\mathrm{OR}$ & $95 \% \mathrm{Cl}$ & $\mathrm{OR}$ & $95 \% \mathrm{Cl}$ \\
\hline Physical & 1.13 & $0.94-1.36$ & $1.45^{\star}$ & * $1.06-1.97$ * & 1.20 & $0.99-1.45$ \\
\hline Mental & 1.02 & $0.88-1.19$ & 0.98 & $0.70-1.37$ & 1.02 & $0.83-1.24$ \\
\hline
\end{tabular}

Table 6. Crude odds ratios (OR) and $95 \%$ confidence intervals $(95 \% \mathrm{Cl})$ for the risk factors in relation to the onset of low-back pain - univariate conditional logistic regression analyses of data with time lag (longitudinal). ${ }^{\text {a }}$

\begin{tabular}{|c|c|c|c|c|c|c|}
\hline \multirow[t]{2}{*}{ Risk factor } & \multicolumn{2}{|c|}{$\begin{array}{l}\text { Low onset } 1 \\
(\mathrm{~N}=81)\end{array}$} & \multicolumn{2}{|c|}{$\begin{array}{l}\text { Medium onset } 2 \\
(\mathrm{~N}=36)\end{array}$} & \multicolumn{2}{|c|}{$\begin{array}{l}\text { High onset } \geq 3 \\
(\mathrm{~N}=44)\end{array}$} \\
\hline & $\mathrm{OR}$ & $95 \% \mathrm{Cl}$ & $\mathrm{OR}$ & $95 \% \mathrm{Cl}$ & $\mathrm{OR}$ & $95 \% \mathrm{Cl}$ \\
\hline me pr & 0.95 & $0.83-1.07$ & 0.84 & $0.69-1.03$ & 1.00 & 0.86 \\
\hline Stress & 0.94 & $0.83-1.07$ & 0.98 & $0.80-1.21$ & 0.89 & $0.72-1.09$ \\
\hline Help & 0.90 & $0.77-1.05$ & 1.19 & $0.97-1.47$ & 1.15 & $0.98-1.36$ \\
\hline Appreciation & 0.99 & $0.85-1.16$ & 1.11 & $0.85-1.45$ & 0.91 & $0.72-1.14$ \\
\hline Conscience & 1.16 & $0.95-1.42$ & 1.09 & $0.80-1.48$ & 1.22 & $0.91-1.64$ \\
\hline Physical exertion & 0.90 & $0.77-1.06$ & 1.03 & $0.81-1.30$ & $0.82^{*}$ & ${ }^{\star} 0.69-0.98^{*}$ \\
\hline Mental exertion & $0.85^{*}$ & * $0.72-1.00$ * & 0.89 & $0.69-1.14$ & 0.95 & $0.75-1.21$ \\
\hline
\end{tabular}

a Number of observations: 243 for onset 1, 108 for onset 2, and 132 for onset $\geq 3$.

${ }^{*} \mathrm{P}<0.05$.

importance of physical and mental exertion. The results in table 5 indicate that the odds ratios and confidence intervals, regardless of onset magnitude, remained rather stable.

\section{Data with a time lag}

None of the odds ratios presented in table 6 support the hypothesis that any of the independent variables can predict low-back pain onset the following day. On the contrary, the results surprisingly included significant odds ratios of 0.82 (95\% CI 0.69-0.98) for physical exertion onset $\geq 3$ and 0.85 (95\% CI 0.72-1.00) for mental exertion onset 1 , a finding which provocatively indicates these to be preventive factors in relation to pain onset.

\section{Discussion}

The main finding of this study was that psychosocial and physical strain on one workday does not predict low- back pain onset the following day. The time-lagged analyses thus failed to support the hypothesized causal relations.

Physical exertion was consistently associated with pain onset on the same day, although it was only significantly related to the two highest levels of pain onset. As in previous studies $(6,7,9-11)$, an association was seen between stress and pain in this study, however only in relation to the most severe pain onset. Modest support for an association between time pressure and a high level of pain onset was also found. This finding may reflect confounding with stress, as indicated by a strong correlation $(\mathrm{r}=0.72)$ between these variables. Exposure and pain seemed to be covariates, as the level of exposure, like pain onset, increased from one day to the next for the cases, while it remained stable for the reference observations. Therefore, causality cannot be inferred from the analyses of data without time lag. However, the design of this study allowed two additional inferences.

First, the different results for the analyses with and without time lag have implications for estimating the effect period of work-related risk factors. The effect period is defined as the period of altered risk in a population, observed as the difference between the minimum delay before impact and the maximum carryover time (20). In this study the effect period, if any effect at all, was less than 24 hours, and measurements should therefore be collected with shorter intervals (eg, every hour during a workday). Obviously, the alternative interpretation is that the minimum delay is more than 24 hours, which however seems unlikely in relation to the acute onset or aggravation of pain.

Second, the case-crossover design of this study eliminated the risk that significant associations would be spuriously inflated by stable subjective characteristics $(19,20)$. Confounding by constant subject characteristics such as age, seniority, smoking, former low-back pain problems, and negative affectivity (a general dimension of subjective distress) (25) was ruled out, as each subject served as her own reference. However, the case-crossover design did not adjust for subject characteristics and environmental factors that may have changed over time [eg, training, organizational structure and new clients (20)]. Furthermore, it is still possible that pain reporting and perceptions of the work environment reflected changing, temporary mental states and consequently that we did not measure two independent phenomena.

\section{Relative importance of physical and mental exertion}

In the analyses without time lag, physical exertion was significantly associated with pain onset (OR 1.13, 1.43, 
and 1.20), while a consistent, but weak positive association was indicated for mental exertion (OR 1.04, 1.08, and 1.06). Only the odds ratios for mental exertion (1.02, 0.98 , and 1.02 ) were slightly affected by entering both factors into a bivariate analysis. These results confirm the importance of physical exertion when compared with an uncertain effect of mental strain in relation to pain onset, although the methodological limitations of this study warrant that the results be interpreted with caution.

First, self-reported physical exertion is likely to be confounded by mental factors (3), as indicated in our study by a correlation between physical and mental exertion ( $\mathrm{r}=0.54)$. Likewise, Josephson et al (18) also reported physical exertion (RPE scale) to be correlated with psychological demands $(\mathrm{r}=0.4)$.

Furthermore, it is puzzling that total mental exertion did not even approach a significant association with pain onset, while stress and time pressure did. Evidently the measure of mental exertion is likely to be confounded by physical exertion, but it is also a limitation of this study that the applied single-item measures provided less valid and reliable data than multiple item scales might have offered. For instance, it is uncertain whether the concept of stress is appraised and reported as a stressor or a response (26).

In summary, in our attempts to determine the relative importance of physical and mental exertion, it was an advantage of our study that comparable self-reported measures were available. However, it was revealed that simply posing similar questions and applying identical scales for each factor side-by-side has methodological limitations $(1,2)$. Perceived exertion may in reality be impossible to separate into distinctive physical and mental components (11), partly because they are indistinguishable for the person, and partly because exertion is an integrated phenomenon.

\section{Selection of reference observations}

Considerable declines in pain - negative onsets were excluded from the reference observations, as they were likely to be derived predominantly from high outset levels of pain, when a decline in pain was to be expected and further increase was unlikely. Furthermore, congruence in the actual low-back pain level at the day of outset had to be observed for the case and reference observations. Otherwise, although neutral in respect of onset, continuous high levels of reported pain were likely to become reference observations.

Reference observations did not necessarily precede the case observations, and this result implies that a case of pain onset may have affected how psychosocial factors and physical exertion were reported in subsequent reference observations. However, we assumed that, when compared with, for example, incidents of severe illness, an episode of acute low-back pain onset had limited effect on later reports.

The aim of this study was to investigate immediate effects of short-term daily psychosocial and physical exposures at work on pain onset. The results should not be extended beyond this perspective without caution. Possible effects of accumulated psychosocial and physical exertion, as well as complex psychological constructs (eg, personality traits or burnout) cannot be ruled out on the basis of this study (21).

\section{Concluding remarks}

In this study we found that psychosocial strain and physical exertion from one day of work did not predict onset of low-back pain the following day. Physical exertion, stress, and possibly time pressure in this care work context were related to acute pain onset the same day, but the associations were weak and rather inconsistent. Thus the short-term effect, if any, has a delay of less than 24 hours.

\section{Acknowledgments}

This study was supported by grants from the Danish Work Environment Fund, Health Funds of the National Health Service of Denmark and the Health Research Campaign of the County of Aarhus.

\section{References}

1. Lagerström M, Hansson T, Hagberg M. Work-related lowback problems in nursing [review]. Scand J Work Environ Health 1998;24:449-64.

2. Josephson M, Vingård E, MUSIC-Norrtälje Study Group. Workplace factors and care seeking for low-back pain among female nursing personnel. Scand J Work Environ Health 1998;24:465-72.

3. Bongers PM, de Winter CR, Kompier MAJ, Hildebrandt VH. Psychosocial factors at work and musculoskeletal disease [review]. Scand J Work Environ Health 1993;19: 297-312.

4. Burdorf A, Sorock GS. Positive and negative evidence of risk factors for back disorders [review]. Scand J Work Environ Health 1997;23:243-56.

5. Hoogendoorn WE, Poppel MNM, Bongers PM, Koes BW, Bouter LM. Systematic review of psychosocial factors at work and private life as risk factors for back pain. Spine 2000;25:2114-25.

6. Bru E, Svebak S, Mykletun R, Gitlesen JP. Back pain, dysphoric versus euphoric moods and the experience of stress and effort in female hospital staff. Pers Individ Differ 1997;22:565-73.

7. Elovainio M, Sinervo T. Psychosocial stressors at work, psychological stress and musculoskeletal symptoms in the 
care for the elderly. Work Stress 1997;11:351-61.

8. Smedley J, Egger P, Cooper C, Coggon D. Manual handling activities and risk of low back pain in nurses. Occup Environ Med 1995;52:160-3.

9. Ahlberg-Hultén GK, Theorell T, Sigala F. Social support, job strain and musculoskeletal pain among female health care personnel. Scand J Work Environ Health 1995; 21:435-9.

10. Josephson M, Lagerström M, Hagberg M, Wigaeus Hjelm E. Musculoskeletal symptoms and job strain among nursing personnel: a study over a three year period. Occup Environ Med 1997;54:681-5.

11. Josephson M. Work factors and musculoskeletal disorders: an epidemiological approach focusing on female nursing personnel. Stockholm: National Institute for Working Life, 1998. Arbete och Hälsa, 1998:30.

12. Lagerström M, Wenemark M, Hagberg M, Wigaeus Hjelm E. Occupational and individual factors related to musculoskeletal symptoms in five body regions among Swedish nursing personnel. Int Arch Occup Environ Health 1995; 68:27-35.

13. Engels JA, Gulden JWJ, Senden TF, Hof B. Work related risk factors for musculoskeletal complaints in the nursing profession: results of a questionnaire survey. Occup Environ Med 1996;53:636-41.

14. Moens GF, Dohogne T, Jacques P, Helshoecht P. Back pain and its correlates among workers in family care. Occup Med 1993;43:78-84.

15. Johansson JÅ. Psychosocial work factors, physical work load and associated musculoskeletal symptoms among home care workers. Scand J Psychol 1995;36:113-29.

16. Bru E, Mykletun RJ, Svebak S. Work-related stress and musculoskeletal pain among female hospital staff. Work Stress
1996;10:309-21.

17. Smedley J, Egger P, Cooper C, Coggon D. Prospective cohort study of predictors of incident low back pain in nurses. BMJ 1997;314:1225-9.

18. Josephson M, Hagberg M, Wigaeus Hjelm E. Self-reported physical exertion in geriatic care. Spine 1996;21:2781-5.

19. Rothman KJ, Greenland S. Case-Control Studies. In: Rothman KJ, Greenland S, editors. Modern epidemiology. Philadelphia (PA): Lippincott-Raven Publishers, 1998:93-114.

20. Maclure M. The case-crossover design: a method for studying transient effects on the risk of acute events. Am J Epidemiol 1991;133:144-51.

21. Riihimäki H. Musculoskeletal diseases - a continuing challenge for epidemiologic research. Scand J Work Environ Health 1999;25 suppl 4:31-5.

22. Hagberg M. Design of intervention studies to improve the health of health care personnel. In: Wickström G, editor. Intervention studies in the health care work environment: lessons learned. Stockholm: Arbetslivsinstitutet, 2000. Arbete och Hälsa 2000;10:13-20.

23. Borg G. Psychophysical scaling with applications in physical work and the perception of exertion. Scand J Work Environ Health 1990;16:55-8.

24. SAS Institute. SAS/STAT user's guide, version 6. 4th ed. Cary (NC): SAS Institute, 1990.

25. Watson D, Pennebaker JW. Health complaints, stress and distress: exploring the central role of negative affectivity. Psychol Rev 1989;96:234-54.

26. Jex JM, Beehr TA, Roberts CK. The meaning of occupational stress items to survey respondents. J Appl Psychol 1992;77:623-8.

Received for publication: 17 April 2001 